\title{
Noncoherent Data Transition Tracking Loops for Symbol Synchronization in Digital Communication Receivers
}

\author{
Marvin K. Simon, Life Fellow, IEEE, and Andre Tkacenko, Member, IEEE
}

\begin{abstract}
Starting with the maximum a posteriori (MAP) estimation approach, this paper derives the optimum (in the MAP estimation sense) means for performing symbol-timing recovery in the absence of carrier-phase information (i.e., prior to carrier-phase recovery). Specifically, we examine the necessary modification of a well-known form of coherent symbol synchronizer, namely, the data transition tracking loop (DTTL), to allow its operation in the absence of carrier-phase information, i.e., as a so-called noncoherent symbol sync loop. By employing such a noncoherent scheme, one can eliminate the need for iteration between the carrier and symbol sync functions, as typically takes place in receivers that more commonly perform carrier tracking and acquisition prior to symbol timing. The performance of both the linear and nonlinear versions of this noncoherent DTTL is obtained by a combination of analysis and simulation, and compared with that of the corresponding coherent DTTLs.
\end{abstract}

Index Terms-Data transition tracking loop (DTTL), noncoherent symbol sync loop, symbol synchronization.

\section{INTRODUCTION}

$\mathbf{T}$ RADITIONAL methods for establishing symbol synchronization (sync) in digital communication receivers assume that carrier sync has already been established, ${ }^{1}$ i.e., the problem is addressed at the baseband level, assuming that a "perfect" estimate of carrier phase is available. We refer to this approach as coherent symbol sync. Since, for nonreturn-to-zero (NRZ) signaling, a suppressed carrier sync loop such as an I-Q Costas loop includes integrate-and-dump (I\&D) filters in its in-phase (I) and quadrature (Q) arms, the traditional approach is to first track the carrier in the absence of symbol sync information, then feed back the symbol sync estimate to these filters, and then iterate between the two to a desirable operating level.

Paper approved by C. Tepedelenlioglu, the Editor for Transmission Systems of the IEEE Communications Society. Manuscript received July 13, 2005; revised October 12, 2005. This work was performed at the Jet Propulsion Laboratory, California Institute of Technology, under a contract with the National Aeronautics and Space Administration.

The authors are with the Jet Propulsion Laboratory, California Institute of Technology, Pasadena, CA 91109 USA (e-mail: marvin.k.simon@jpl.nasa.gov; andre.tkacenko@jpl.nasa.gov).

Digital Object Identifier 10.1109/TCOMM.2006.873984

\footnotetext{
${ }^{1}$ The ability to obtain carrier sync prior to symbol sync is enabled by the possibility of successfully operating a Costas loop with I\&D arm filters in the absence of symbol sync knowledge. Even in the worst case of a symbol sync error equal to one-half a data-symbol time interval, the loop still provides an estimate of carrier phase, albeit at an increased mean-square phase error. On the contrary, a conventional symbol sync scheme cannot guarantee performing its function in the absence of carrier-phase knowledge, particularly in the worst case of a phase error equal to $\pi / 2$ rads.
}

In this paper, we investigate means for performing symboltiming recovery prior to carrier-phase recovery. In particular, we reexamine a well-known form of coherent symbol synchronizer, namely, the data transition tracking loop (DTTL), with emphasis on modifying it to operate in the absence of carrier-phase information, i.e., as a so-called noncoherent symbol sync loop. By employing such a noncoherent scheme, we can eliminate the need for iteration between the carrier and symbol sync functions. Although originally proposed as an ad hoc scheme back in the late 1960s [1], [6], it was later shown (in unpublished work) that by suitably approximating the derivative of the rectangular symbol pulse shape, the DTTL can be motivated by the maximum a posteriori (MAP) approach to symbol-timing estimation. In arriving at the necessary modifications for noncoherent operation, as mentioned above, we shall employ a MAP motivation similar to that previously used in arriving at the phase-coherent DTTL. Finally, we compare the performance of the high and low signal-to-noise ratio (SNR) versions of the noncoherent DTTL (NC-DTTL) with their coherent counterparts. The performance results will be obtained from a combination of analysis and computer simulation.

It should be noted that the MAP approach to symbol sync was considered in [2], in the context of arriving at a nondata-aided recursive algorithm for symbol timing. Although, at first glance, it might appear that the approach taken there corresponds to noncoherent symbol sync, since the carrier phase was assumed to be unknown but independent from symbol to symbol, ${ }^{2}$ in reality, the derivation of the MAP estimate of symbol sync was preceded by a recursive estimate of the carrier phase, which justifies such an assumption. Our emphasis here, as mentioned above, is on interpreting the likelihood function (LF) derived from such an approach in such a way as to arrive at noncoherent versions of the DTTL. For convenience, we shall adopt a similar notation to that used in [2], so that the reader can follow both approaches and discern the differences.

\section{MAP SyMBol SynC ESTIMATION IN THE AbSENCE OF CARRIER-PHASE INFORMATION}

\section{A. Derivation of the Exact $L F$}

The input to the receiver is a binary phase-shift keying (BPSK) modulation consisting of a binary NRZ datastream

\footnotetext{
${ }^{2}$ As we shall see shortly, the appropriate assumption for truly noncoherent symbol sync is an unknown carrier phase that is constant over the duration of the observation, i.e., a sequence of symbols.
} 
direct-modulated onto a carrier with known frequency and unknown phase plus noise, and is modeled as

$x(t)=\sqrt{2 P}\left(\sum_{k=-\infty}^{\infty} d_{k} p(t-(k-1+\varepsilon) T)\right) \cos \left(\omega_{c} t+\theta_{c}\right)+n(t)$

where $P$ is the total received power in the signal, $\omega_{c}$ and $\theta_{c}$ are the carrier radian frequency and phase, $p(t)$ is a unit amplitude rectangular pulse shape of duration $T$ seconds, $\left\{d_{n}\right\}$ is the data sequence consisting of independent identically distributed (i.i.d.) equiprobable \pm 1 data symbols, and $0 \leq \varepsilon \leq 1$ is the fractional timing offset to be estimated. Also in (1), $n(t)$ is a bandpass additive white Gaussian noise (AWGN) process that can be represented as

$$
n(t)=\sqrt{2}\left[N_{c}(t) \cos \left(\omega_{c} t+\theta_{c}\right)-N_{s}(t) \sin \left(\omega_{c} t+\theta_{c}\right)\right]
$$

with $N_{c}(t), N_{s}(t)$ lowpass AWGN processes with single-sided power spectral density (PSD) $N_{0} \mathrm{~W} / \mathrm{Hz}$.

The first step is to demodulate the received signal with the quadrature carrier reference signals

$$
r_{c}(t)=\sqrt{2} \cos \omega_{c} t, \quad r_{s}(t)=-\sqrt{2} \sin \omega_{c} t
$$

resulting in the pair of baseband observables in the $n$th symbol interval $(n-1+\varepsilon) T \leq t \leq(n+\varepsilon) T$

$$
\begin{aligned}
x_{c n}(t)= & \sqrt{P} d_{n} p(t-(n-1+\varepsilon) T) \cos \theta_{c}+N_{c}(t) \cos \theta_{c} \\
& -N_{s}(t) \sin \theta_{c} \\
= & s\left(t, \varepsilon, d_{n}\right) \cos \theta_{c}+n_{c n}\left(t, \theta_{c}\right) \\
x_{s n}(t)= & \sqrt{P} d_{n} p(t-(n-1+\varepsilon) T) \sin \theta_{c}+N_{c}(t) \sin \theta_{c} \\
& +N_{s}(t) \cos \theta_{c} \\
= & s\left(t, \varepsilon, d_{n}\right) \sin \theta_{c}+n_{s n}\left(t, \theta_{c}\right) .
\end{aligned}
$$

Since $x_{c n}(t)$ and $x_{s n}(t)$ represent the received signal at baseband, it is convenient to express them in the complex form as

$$
\tilde{x}_{n}(t)=x_{c n}(t)+j x_{s n}(t)=s\left(t, \varepsilon, d_{n}\right) e^{j \theta_{c}}+\tilde{N}_{n}\left(t, \theta_{c}\right)
$$

where

$$
\begin{aligned}
\tilde{N}_{n}(t, \theta) & =n_{c n}(t)+j n_{s n}(t)=\tilde{N}_{n}(t) e^{j \theta_{c}} \\
\tilde{N}_{n}(t) & =N_{c}(t)+j N_{s}(t) .
\end{aligned}
$$

Then, for an observation of duration NT seconds, corresponding to $N$ i.i.d. symbols, the joint probability density function (pdf) of the collection of complex observables $\tilde{x}(t)=\left(\tilde{x}_{1}(t), \tilde{x}_{2}(t), \ldots, \tilde{x}_{N}(t)\right)$, conditioned on the transmitted symbol vector $\mathbf{d}=\left[d_{1}, d_{2}, \ldots, d_{N}\right]$ and the occurrence of the unknown carrier phase $\theta_{c}$ and fractional symbol-timing offset $\varepsilon_{r}$, is given by

$$
\begin{aligned}
p_{\mathbf{x}} & \left(\tilde{x}(t) \mid \mathbf{d}, \varepsilon, \theta_{c}\right) \\
& =\frac{1}{\pi N_{0}} \exp \left(-\frac{1}{N_{0}} \int_{T_{o}(\varepsilon)}\left\|\tilde{x}(t)-s(t, \varepsilon, \mathbf{d}) e^{j \theta_{c}}\right\|^{2} d t\right) \\
& =C \exp \left(\frac{2}{N_{0}} \Re\left\{\int_{T_{o}(\varepsilon)} \tilde{x}(t) \cdot s(t, \varepsilon, \mathbf{d}) e^{-j \theta_{c}} d t\right\}\right)
\end{aligned}
$$

where $T_{o}(\varepsilon)$ denotes the observation time interval $\varepsilon T \leq t \leq$ $(N+\varepsilon) T$, and $C$ is a constant that is independent of the unknown parameters, and also reflects the constant energy nature of the BPSK modulation. The pdf in (7) is traditionally referred to as a conditional $\mathrm{LF}(\mathrm{CLF})$, and given the notation $L\left(\mathbf{d}, \varepsilon, \theta_{c}\right)$.

Because of the i.i.d. property of the data symbols, the CLF can be expressed as the product of per-symbol CLFs, namely

$$
\begin{aligned}
& L\left(\mathbf{d}, \varepsilon, \theta_{c}\right) \\
& =\prod_{n=1}^{N} \exp \left(\frac{2}{N_{0}} \Re\left\{\int_{T_{n}(\varepsilon)} \tilde{x}_{n}(t) s\left(t, \varepsilon, d_{n}\right) e^{-j \theta_{c}} d t\right\}\right)
\end{aligned}
$$

where $T_{n}(\varepsilon)$ denotes the time interval $(n-1+\varepsilon) T \leq t \leq$ $(n+\varepsilon) T$, and, for simplicity, we have ignored all multiplicative constants, since they do not affect the parameter estimation.

The issue that arises now is the order in which to perform the averaging over the unknown data sequence and the unknown carrier phase. Suppose that one attempts to first average over the carrier phase. In order to do this, we rewrite (8) in the form

$$
\begin{aligned}
& L\left(\mathbf{d}, \varepsilon, \theta_{c}\right) \\
& \quad=\exp \left(\frac{2}{N_{0}} \Re\left\{\sum_{n=1}^{N} \int_{T_{n}(\varepsilon)} \tilde{x}_{n}(t) s\left(t, \varepsilon, d_{n}\right) e^{-j \theta_{c}} d t\right\}\right) \\
& \quad=\exp \left\{\frac{2}{N_{0}} R(\mathbf{d}, \varepsilon) \cos \left[\theta_{c}-\alpha(\mathbf{d}, \varepsilon)\right]\right\}
\end{aligned}
$$

where

$$
\begin{aligned}
R(\mathbf{d}, \varepsilon) & =\left|\sum_{n=1}^{N} \int_{T_{n}(\varepsilon)} \tilde{x}_{n}(t) s\left(t, \varepsilon, d_{n}\right) d t\right| \\
& =\left|\sum_{n=1}^{N} d_{n} \sqrt{P} \int \tilde{x}_{n}(t) p(t-(n-1+\varepsilon) T) d t\right| \\
\alpha(\mathbf{d}, \varepsilon) & =\arg \left\{\sum_{n=1}^{N} \int_{T_{n}(\varepsilon)} \tilde{x}_{n}(t) s\left(t, \varepsilon, d_{n}\right) d t\right\} .
\end{aligned}
$$

Averaging over the uniformly distributed carrier phase we get ${ }^{3}$

$$
\begin{aligned}
L(\mathbf{d}, \varepsilon) & =I_{0}\left(\frac{2}{N_{0}} R(\mathbf{d}, \varepsilon)\right) \\
& =I_{0}\left(\frac{2 \sqrt{P}}{N_{0}}\left|\sum_{n=1}^{N} d_{n} \int_{T_{n}(\varepsilon)} \tilde{x}_{n}(t) p(t-(n-1+\varepsilon) T) d t\right|\right)
\end{aligned}
$$

where $I_{0}(x)$ is the zero-order modified Bessel function of the first kind with argument $x$.

The difficulty now lies in analytically averaging over the data sequence in (11) when $N$ is large. Thus, in order to arrive at simple metrics, before averaging over the data, we must first

${ }^{3}$ At this point, it should be reemphasized that our approach differs from that in [2] in that in the latter, the per-symbol LF is averaged over the carrier phase, and then, because of the i.i.d. nature of the data, an LF is formed from the product of these phase-averaged LFs. Forming the LF in such a way implicitly assumes that the carrier phase varies independently from symbol to symbol, which is in opposition to our assumption that the carrier phase is constant over the observation. 


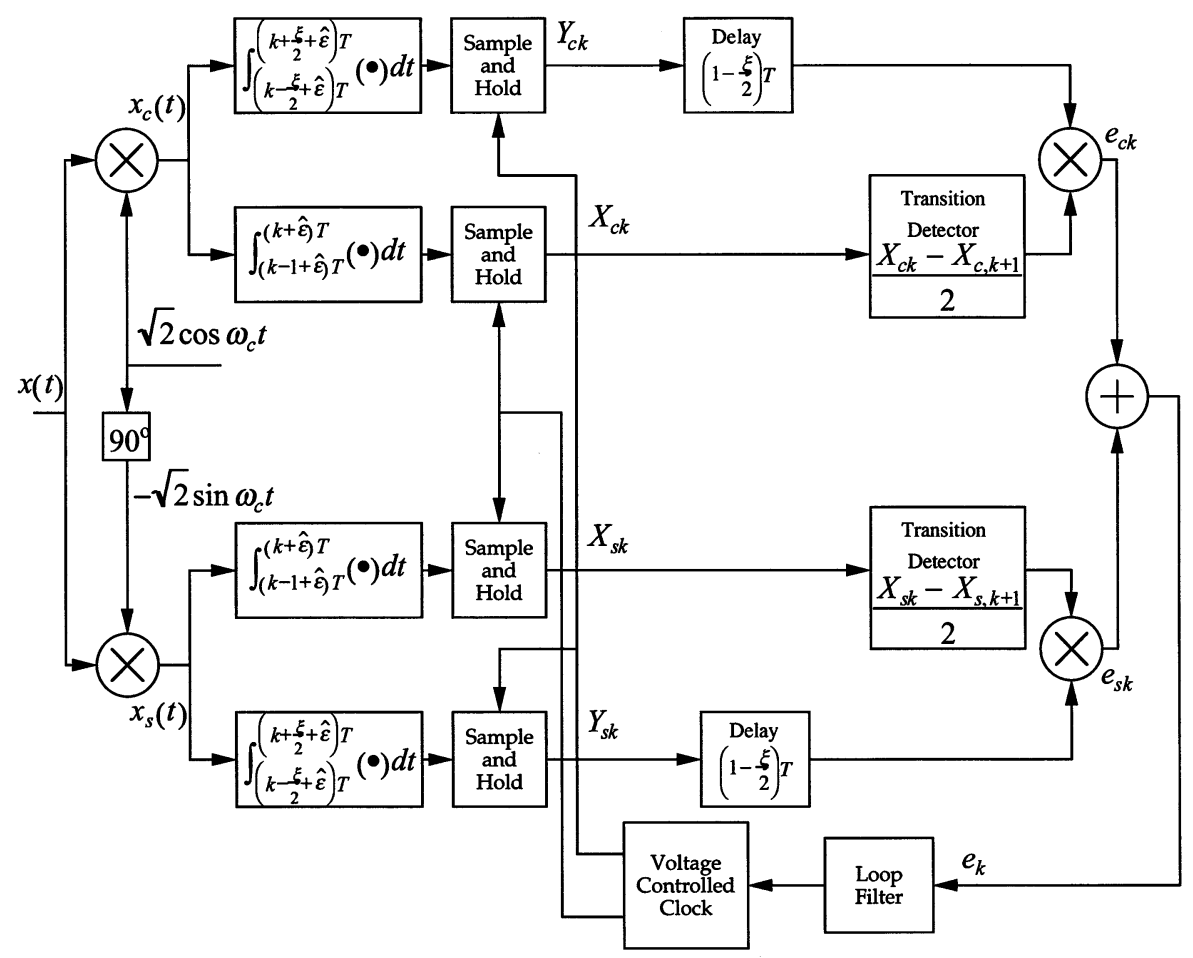

Fig. 1. NC-LDTTL.

simplify matters by approximating the nonlinear (Bessel) function in (11).

\section{B. Low SNR Approximation of the CLF-NC-LDTTL}

Since the argument of the Bessel function in (11) is proportional to $P T / N_{0}$, then for low SNR, the following approximation is appropriate:

$$
I_{0}(x) \cong 1+\frac{x^{2}}{4} .
$$

Applying (12) to (11) and defining the real observables

$$
\begin{aligned}
& X_{c n}(\varepsilon) \triangleq \int_{T_{n}(\varepsilon)} x_{c n}(t) p(t-(n-1+\varepsilon) T) d t \\
& X_{s n}(\varepsilon) \triangleq \int_{T_{n}(\varepsilon)} x_{s n}(t) p(t-(n-1+\varepsilon) T) d t
\end{aligned}
$$

we obtain

$$
\begin{aligned}
L(\mathbf{d}, \varepsilon) & =I_{0}\left(\frac{2 \sqrt{P}}{N_{0}}\left|\sum_{n=1}^{N} d_{n}\left(X_{c n}(\varepsilon)+j X_{s n}(\varepsilon)\right)\right|\right) \\
& \cong 1+\frac{P}{N_{0}^{2}}\left|\sum_{n=1}^{N} d_{n}\left(X_{c n}(\varepsilon)+j X_{s n}(\varepsilon)\right)\right|^{2} \\
& =1+\frac{P}{N_{0}^{2}}\left|\sum_{n=1}^{N} d_{n} X_{c n}(\varepsilon)+j \sum_{n=1}^{N} d_{n} X_{s n}(\varepsilon)\right|^{2} \\
& =1+\frac{P}{N_{0}^{2}}\left[\left(\sum_{n=1}^{N} d_{n} X_{c n}(\varepsilon)\right)^{2}+\left(\sum_{n=1}^{N} d_{n} X_{s n}(\varepsilon)\right)^{2}\right] .
\end{aligned}
$$

Finally, averaging over the i.i.d. data sequence gives the simplified LF

$$
L(\varepsilon)=1+\frac{P}{N_{0}^{2}} \sum_{n=1}^{N} X_{c n}^{2}(\varepsilon)+\frac{P}{N_{0}^{2}} \sum_{n=1}^{N} X_{s n}^{2}(\varepsilon) .
$$

To arrive at a closed-loop symbol sync structure motivated by this LF, we proceed in the usual way by differentiating the LF with respect to $\varepsilon$ and using the result to form the error signal in the loop. Taking the partial derivative of (16) with respect to $\varepsilon$, and again ignoring multiplicative constants, gives

$$
\frac{\partial L(\varepsilon)}{\partial \varepsilon}=\sum_{n=1}^{N}\left[X_{c n}(\varepsilon) \frac{d X_{c n}(\varepsilon)}{d \varepsilon}+X_{s n}(\varepsilon) \frac{d X_{s n}(\varepsilon)}{d \varepsilon}\right]
$$

each of whose terms is analogous to that which forms the error signal in the low-SNR version of the coherent DTTL (referred to as a linear DTTL (LDTTL) in [3]). Thus, the low-SNR version of the NC-DTTL, herein given the acronym NC-LDTTL, is nothing more than the parallel combination of two coherent LDTTLs acting on the I and Q baseband signals. A block diagram of this structure is illustrated in Fig. 1, and the analysis of its performance will follow in the next section.

\section{High-SNR Approximation of the CLF}

For large SNR, we need to approximate $I_{0}(x)$ in (11) by its large argument form, which behaves as $\exp (|x|)$. Thus, in this case, the CLF would be approximated as

$$
\begin{aligned}
& L(\mathbf{d}, \varepsilon) \cong \\
& \quad \exp \left(\frac{2 \sqrt{P}}{N_{0}}\left|\sum_{n=1}^{N} d_{n} \int_{T_{n}(\varepsilon)} \tilde{x}_{n}(t) p(t-(n-1+\varepsilon) T) d t\right|\right)
\end{aligned}
$$


which unfortunately does not ease the burden of averaging over the data sequence.

Suppose now instead we first average the CLF over the data. Then from (8) we have (18), shown at the bottom of the page, where

$$
\beta_{n}=\tan ^{-1} \frac{X_{s n}(\varepsilon)}{X_{c n}(\varepsilon)} .
$$

If we now apply the large argument (e.g., high SNR) approximation to the hyperbolic cosine function, namely

$$
\cosh x \cong \frac{\exp (|x|)}{2}
$$

then (18) becomes (ignoring multiplicative constants)

$$
\begin{aligned}
& L\left(\varepsilon, \theta_{c}\right)= \\
& \quad \exp \left\{\sum_{n=1}^{N}\left|\frac{2 \sqrt{P}}{N_{0}} \sqrt{\left(X_{c n}(\varepsilon)\right)^{2}+\left(X_{s n}(\varepsilon)\right)^{2}} \cos \left(\theta_{c}-\beta_{n}\right)\right|\right\}
\end{aligned}
$$

which still presents difficulty in analytically averaging over the unknown parameter, in this case, $\theta_{c}$. Thus, having failed on both attempts at averaging the CLF over both the carrier phase and the data sequence at high SNR, we are forced to deviate from the true maximum-likelihood (ML) approach in favor of one that will provide a simple metric.

\section{Suboptimum Approach Based on an Ad Hoc Approximation} to the Generalized Likelihood Ratio-Noncoherent Nonlinear DTTL (NC-NLDTTL)

Another approach, albeit suboptimum, that can achieve near-ML performance is to choose (rather than average over) the value of the unknown parameter that maximizes the CLF. This is commonly referred to as a generalized-likelihood ratio approach. With reference to (11), in the limit of no noise (infinite SNR), the CLF $L(\mathbf{d}, \varepsilon)$, or equivalently, the argument of the Bessel function, would be maximized when

$$
d_{n}=\operatorname{sgn}\left[\int_{T_{n}(\varepsilon)} s\left(t, \varepsilon, d_{n}\right) p(t-(n-1+\varepsilon) T) d t\right]
$$

i.e., all the signal vectors are aligned in the same direction, in which case, the argument of the Bessel function (ignoring the constant multiplicative factor) would become

$$
\begin{aligned}
& \left|\sum_{n=1}^{N} d_{n} \int_{T_{n}(\varepsilon)} s\left(t, \varepsilon, d_{n}\right) e^{j \theta_{c}} p(t-(n-1+\varepsilon) T) d t\right| \\
& \quad=\sum_{n=1}^{N}\left|\int_{T_{n}(\varepsilon)} s\left(t, \varepsilon, d_{n}\right) e^{j \theta_{c}} p(t-(n-1+\varepsilon) T) d t\right| .
\end{aligned}
$$

Thus, as a high-SNR approximation of this limiting case, we propose the ad hoc unconditional LF

$$
L(\varepsilon)=I_{0}\left(\frac{2 \sqrt{P}}{N_{0}} \sum_{n=1}^{N}\left|\int_{T_{n}(\varepsilon)} \tilde{x}_{n}(t) p(t-(n-1+\varepsilon) T) d t\right|\right)
$$

or equivalently, taking the natural logarithm of (23), the loglikelihood function (LLF)

$$
\begin{aligned}
l(\varepsilon) & =\ln L(\varepsilon) \\
& =\ln I_{0}\left(\frac{2 \sqrt{P}}{N_{0}} \sum_{n=1}^{N}\left|\int_{T_{n}(\varepsilon)} \tilde{x}_{n}(t) p(t-(n-1+\varepsilon) T) d t\right|\right) \\
& =\ln I_{0}\left(\frac{2 \sqrt{P}}{N_{0}} \sum_{n=1}^{N}\left|X_{c n}(\varepsilon)+j X_{s n}(\varepsilon)\right|\right) \\
& =\ln I_{0}\left(\frac{2 \sqrt{P}}{N_{0}} \sum_{n=1}^{N} \sqrt{X_{c n}^{2}(\varepsilon)+X_{s n}^{2}(\varepsilon)}\right) .
\end{aligned}
$$

For large arguments, the nonlinearity in (24) can be approximated (to within a scaling constant) as

$$
\ln I_{0}(x) \cong|x|
$$

which after substitution in (24) yields

$$
l(\varepsilon)=\ln L(\varepsilon)=\left|\frac{2 \sqrt{P}}{N_{0}} \sum_{n=1}^{N} \sqrt{X_{c n}^{2}(\varepsilon)+X_{s n}^{2}(\varepsilon)}\right| .
$$

$$
\begin{aligned}
L\left(\varepsilon, \theta_{c}\right) & =\underset{\mathbf{d}}{E}\left\{\prod_{n=1}^{N} \exp \left(\frac{2}{N_{0}} \Re\left\{\int_{T_{n}(\varepsilon)} \tilde{x}_{n}(t) s\left(t, \varepsilon, d_{n}\right) e^{-j \theta_{c}} d t\right\}\right)\right\} \\
& =\prod_{n=1}^{N} \underset{d_{n}}{E}\left\{\exp \left(d_{n} \frac{2 \sqrt{P}}{N_{0}} \Re\left\{\int_{T_{n}(\varepsilon)} \tilde{x}_{n}(t) p(t-(n-1+\varepsilon) T) e^{-j \theta_{c}} d t\right\}\right)\right\} \\
& =\prod_{n=1}^{N} \cosh \left(\frac{2 \sqrt{P}}{N_{0}}\left[X_{c n}(\varepsilon) \cos \theta_{c}+X_{s n}(\varepsilon) \sin \theta_{c}\right]\right) \\
& =\prod_{n=1}^{N} \cosh \left(\frac{2 \sqrt{P}}{N_{0}} \sqrt{\left(X_{c n}(\varepsilon)\right)^{2}+\left(X_{s n}(\varepsilon)\right)^{2}} \cos \left(\theta_{c}-\beta_{n}\right)\right)
\end{aligned}
$$




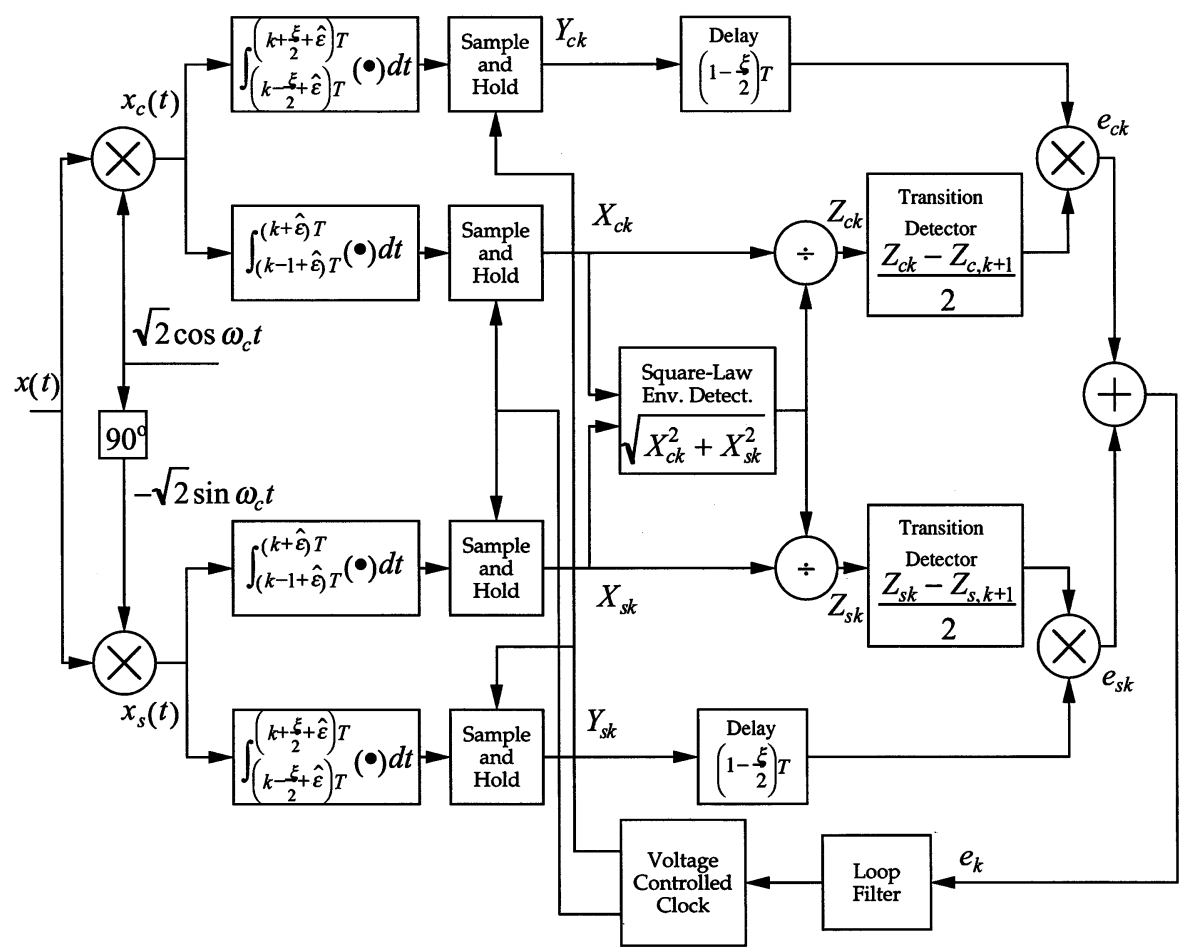

Fig. 2. NC-DTTL

Thus, analogous to (16), differentiating $l(\varepsilon)$ with respect to $\varepsilon$, the error signal in a closed-loop configuration should be formed from

$$
\begin{aligned}
e=\sum_{n=1}^{N}\left[\frac{X_{c n}(\varepsilon)}{\sqrt{X_{c n}^{2}(\varepsilon)+X_{s n}^{2}(\varepsilon)}} \frac{d X_{c n}(\varepsilon)}{d \varepsilon}\right. & \\
& \left.+\frac{X_{s n}(\varepsilon)}{\sqrt{X_{c n}^{2}(\varepsilon)+X_{s n}^{2}(\varepsilon)}} \frac{d X_{s n}(\varepsilon)}{d \varepsilon}\right] .
\end{aligned}
$$

An NC-DTTL-type symbol synchronizer that is motivated by using (27) as its error signal is illustrated in Fig. 2. The analysis of its performance will be discussed later on. In the meantime, it is interesting to note that if one were to consider the coherent case, wherein $X_{s n}(\varepsilon)$ would be absent, then setting $X_{s n}(\varepsilon)$ equal to zero in (27) gives

$$
e=\sum_{n=1}^{N} \frac{X_{c n}(\varepsilon)}{\sqrt{X_{c n}^{2}(\varepsilon)}} \frac{d X_{c n}(\varepsilon)}{d \varepsilon}=\sum_{n=1}^{N} \frac{d X_{c n}(\varepsilon)}{d \varepsilon} \operatorname{sgn} X_{c n}(\varepsilon)
$$

which is exactly the error signal that would be derived from the MAP approach under the assumption of perfectly known carrier phase, and thus motivates the construction of the conventional nonlinear DTTL.
Before proceeding, it is interesting at this point to note that the LLF in (24) is the same ${ }^{4}$ as that given in [2, eq. 6.279], which is obtained by averaging over the carrier phase under the assumption that it varies independently from symbol to symbol, as discussed in footnote 3 . The LLF in (24) can also be obtained from (21) by again assuming that the carrier phase varies independently from symbol to symbol, which is tantamount to replacing $\theta_{c}$ by $\theta_{c n}$ in this equation, and then maximizing over the sequence of carrier phases. It is clear from (21) that this maximization would occur for $\theta_{c n}=\beta_{n}$, in which case, we obtain the LF

$$
L(\varepsilon)=\exp \left\{\sum_{n=1}^{N}\left|\frac{2 \sqrt{P}}{N_{0}} \sqrt{\left(X_{c n}(\varepsilon)\right)^{2}+\left(X_{s n}(\varepsilon)\right)^{2}}\right|\right\} .
$$

Finally, taking the natural logarithm of (29) produces a result identical to (26) for the LLF.

\section{TRacking Performance of THE NC-LDTTL}

With reference to Fig. 1, the upper and lower channels (herein referred to as "cosine" and "sine" channels) inputs to the I\&D filters in the $n$th symbol interval are described by (4). The local clock produces a timing reference for the I and Q I\&D filters of each of these channels, which depends on the estimate $\hat{\varepsilon}$ of $\varepsilon$. As such, the outputs of these same filters are, respectively, given

${ }^{4}$ The only difference is a factor of $\sqrt{T}$ in the argument of the Bessel function, i.e, $2 \sqrt{P} / N_{0}$ in our result, versus $2 \sqrt{E} / N_{0}=2 \sqrt{P T} / N_{0}$ in that of [2], which comes about because of the difference in the normalization of the carrier reference signals between the two approaches. 
by (assuming for simplicity that all gains are set equal to unity) (30) and (31), shown at the bottom of the page.

Since $\mu_{c k}$ and $\nu_{c k}$ are not independent, and likewise for $\mu_{s k}$ and $\nu_{s k}$, it is convenient, as was done in [1], [3], [4], and [6], to express them in terms of a new set of variables

$$
\begin{array}{ll}
\nu_{c k}=N_{c k}+M_{c k}, & \mu_{c k}=M_{c k}^{\prime}+N_{c, k+1}^{\prime} \\
\nu_{s k}=N_{s k}+M_{s k}, & \mu_{s k}=M_{s k}^{\prime}+N_{s, k+1}^{\prime}
\end{array}
$$

where

$$
\begin{aligned}
N_{c k}= & \int_{(k-1+\hat{\varepsilon}) T}^{\left(k-\frac{1}{2}+\hat{\varepsilon}\right) T} N_{c}(t) d t, \quad M_{c k}=\int_{\left(k-\frac{1}{2}+\hat{\varepsilon}\right) T}^{(k+\hat{\varepsilon}) T} N_{c}(t) d t \\
N_{c k}^{\prime}= & \int_{(k-1+\hat{\varepsilon}) T}^{\left(k-1+\frac{\xi}{2}+\hat{\varepsilon}\right) T} N_{c}(t) d t, \quad M_{c k}^{\prime}=\int_{\left(k-\frac{\xi}{2}+\hat{\varepsilon}\right) T}^{(k+\hat{\varepsilon}) T} N_{c}(t) d t
\end{aligned}
$$

with the properties

$N_{c k}, M_{c n} \quad$ mutually independent for all $k, n$

$N_{c k}^{\prime}, M_{c n}^{\prime} \quad$ mutually independent for all $k, n$

$N_{c k}^{\prime}, M_{c n} ; \quad$ mutually independent for all $k, n$

$M_{c k}^{\prime}, N_{c n}$

$N_{c k}^{\prime}, N_{c n}^{\prime} ; \quad$ mutually independent for all $k \neq n$

$M_{c k}, M_{c n}$

Furthermore, all $M_{c k}, M_{c k}^{\prime}, N_{c k}, N_{c k}^{\prime}$ and their sums are Gaussian random variables with zero mean and variances

$$
\sigma_{M_{c k}}^{2}=\sigma_{N_{c k}}^{2}=\frac{N_{0} T}{4}, \quad \sigma_{M_{c k}^{\prime}}^{2}=\sigma_{N_{c k}^{\prime}}^{2}=\frac{\xi N_{0} T}{4} .
$$

Analogous definitions and properties apply to the sine channel noise variables.
Taking the difference of two successive soft decisions $X_{c k}$ and $X_{c, k+1}$ (or $X_{s k}$ and $X_{s, k+1}$ ) and multiplying the average of the result by the quadrature I\&D output $Y_{c k}$ (or $Y_{s k}$ ) delayed by $(1-\xi / 2) T$ gives the sine and cosine channel-error signal components in the $k$ th symbol interval

$$
\begin{gathered}
e_{c k}=\left(b_{k} \cos \theta_{c}+\left(M_{c k}^{\prime}+N_{c, k+1}^{\prime}\right) \cos \theta_{c}-\left(M_{s k}^{\prime}+N_{s, k+1}^{\prime}\right) \sin \theta_{c}\right) \\
\times \frac{1}{2}\left\{\left[c_{k} \cos \theta_{c}+\left(N_{c k}+M_{c k}\right) \cos \theta_{c}\right.\right. \\
\left.-\left(N_{s k}+M_{s k}\right) \sin \theta_{c}\right] \\
-\left[c_{k+1} \cos \theta_{c}+\left(N_{c, k+1}+M_{c, k+1}\right) \cos \theta_{c}\right. \\
\left.\left.-\left(N_{s, k+1}+M_{s, k+1}\right) \sin \theta_{c}\right]\right\}
\end{gathered}
$$

and

$$
\begin{gathered}
e_{s k}=\left(b_{k} \sin \theta_{c}+\left(M_{c k}^{\prime}+N_{c, k+1}^{\prime}\right) \sin \theta_{c}+\left(M_{s k}^{\prime}+N_{s, k+1}^{\prime}\right) \cos \theta_{c}\right) \\
\times \frac{1}{2}\left\{\left[c_{k} \sin \theta_{c}+\left(N_{c k}+M_{c k}\right) \sin \theta_{c}\right.\right. \\
\left.+\left(N_{s k}+M_{s k}\right) \cos \theta_{c}\right] \\
-\left[c_{k+1} \sin \theta_{c}+\left(N_{c, k+1}+M_{c, k+1}\right) \sin \theta_{c}\right. \\
\left.\left.+\left(N_{s, k+1}+M_{s, k+1}\right) \cos \theta_{c}\right]\right\} .
\end{gathered}
$$

The total error signal $e_{k}$ is the sum of the two components in (35) and (36).

\section{A. S-Curve Performance}

The S-curve is by definition the statistical average of the error signal over the signal and noise probability distributions. Letting $\lambda \triangleq \varepsilon-\hat{\varepsilon}$ denote the normalized timing error $(-1 / 2 \leq \lambda \leq$ $1 / 2)$, the S-curve $g(\lambda)$ becomes

$$
\begin{aligned}
g(\lambda)= & E_{n, s}\left\{e_{c k}+e_{s k}\right\} \\
= & E_{s}\left\{b_{k}\left(\frac{c_{k}-c_{k+1}}{2}\right) \cos ^{2} \theta_{c}\right. \\
& \left.+b_{k}\left(\frac{c_{k}-c_{k+1}}{2}\right) \sin ^{2} \theta_{c}\right\} \\
= & E_{s}\left\{b_{k}\left(\frac{c_{k}-c_{k+1}}{2}\right)\right\}
\end{aligned}
$$

$$
\begin{aligned}
& X_{c k}=\int_{(k-1+\hat{\varepsilon}) T}^{(k+\hat{\varepsilon}) T} x_{c k}(t) d t=\cos \theta_{c} \overbrace{\int_{(k-1+\hat{\varepsilon}) T}^{(k+\hat{\varepsilon}) T} s\left(t, \varepsilon, d_{k}\right) d t}^{c_{k}}+\cos \theta_{c} \overbrace{\int_{(k-1+\hat{\varepsilon}) T}^{(k+\hat{\varepsilon}) T} N_{c}(t) d t-\sin \theta_{c} \overbrace{\int_{(k-1+\hat{\varepsilon}) T}^{(k+\hat{\varepsilon}) T}}^{\nu_{c k}} N_{s}(t) d t}^{\nu_{s k}} \\
& Y_{c k}=\int_{\left(k-\frac{\xi}{2}+\hat{\varepsilon}\right) T}^{\left(k+\frac{\xi}{2}+\hat{\varepsilon}\right) T} x_{c k}(t) d t=\cos \theta_{c} \overbrace{\int_{\left(k-\frac{\xi}{2}+\hat{\varepsilon}\right) T}^{\left(k+\frac{\xi}{2}+\hat{\varepsilon}\right) T} s\left(t, \varepsilon, d_{k}\right) d t+\cos \theta_{c}}^{b_{k}} \overbrace{\int_{\left(k-\frac{\xi}{2}+\hat{\varepsilon}\right) T}^{\left(k+\frac{\xi}{2}+\hat{\varepsilon}\right) T} N_{c}(t) d t-\sin \theta_{c}}^{\mu_{c k}} \overbrace{\int_{\left(k-\frac{\xi}{2}+\hat{\varepsilon}\right) T}^{\left(k+\frac{\xi}{2}+\hat{\varepsilon}\right) T}}^{\mu_{s k}} N_{s}(t) d t
\end{aligned}
$$

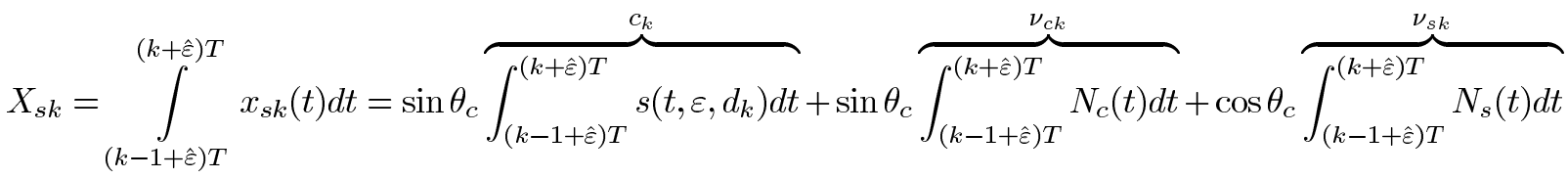

$$
\begin{aligned}
& Y_{s k}=\int_{\left(k-\frac{\xi}{2}+\hat{\varepsilon}\right) T}^{\left(k+\frac{\xi}{2}+\hat{\varepsilon}\right) T} x_{s k}(t) d t=\sin \theta_{c} \overbrace{\int_{\left(k-\frac{\xi}{2}+\hat{\varepsilon}\right) T}^{\left(k+\frac{\xi}{2}+\hat{\varepsilon}\right) T} s\left(t, \varepsilon, d_{k}\right) d t+\sin \theta_{c}}^{b_{k}} \overbrace{\int_{\left(k-\frac{\xi}{2}+\hat{\varepsilon}\right) T}^{\left(k+\frac{\xi}{2}+\hat{\varepsilon}\right) T} N_{c}(t) d t+\cos \theta_{c} \overbrace{\left(k-\frac{\xi}{2}+\hat{\varepsilon}\right) T}^{\left(k+\frac{\xi}{2}+\hat{\varepsilon}\right) T}}^{\mu_{c k}} \overbrace{\int_{s}(t) d t}^{\mu_{s k}}
\end{aligned}
$$


which is independent of the carrier-phase error, as expected, and also identical to the result for the coherent LDTTL. Thus, using the results from [3] with a slight change to the notation used here, we have

$$
g_{n}(\lambda) \triangleq \frac{g(\lambda)}{P T^{2}}= \begin{cases}\lambda\left(1-\frac{\xi}{4}\right)-\frac{3}{2} \lambda^{2}, & 0 \leq \lambda \leq \frac{\xi}{2} \\ \frac{\xi}{2}(1-2 \lambda), & \frac{\xi}{2} \leq \lambda \leq \frac{1}{2} .\end{cases}
$$

As noted in [3], the normalized S-curve for the NC-LDTTL is independent of SNR, whereas that for the conventional (nonlinear) DTTL is highly dependent on SNR.

The slope of the normalized S-curve at the origin $(\lambda=0)$ will be of interest in computing the mean-square timing jitter performance. Taking the derivative of (38) with respect to $\lambda$ and evaluating the result at $\lambda=0$ gives for the NC-LDTTL [3]

$$
\left.K_{g} \triangleq \frac{d g(\lambda)}{d \lambda}\right|_{\lambda=0}=P T^{2}\left(1-\frac{\xi}{4}\right)
$$

\section{B. Noise Performance}

The equivalent noise $n_{\lambda}(t)$ perturbing the loop is characterized by the variation of the loop error signal around its mean (the S-curve), i.e.,

$$
\begin{aligned}
n_{\lambda}(t) & =e_{k}-E_{n, s}\left\{e_{k}\right\} \\
& =e_{k}-g(\lambda), \quad(k+1) T+\hat{\varepsilon} \leq t \leq(k+2) T+\hat{\varepsilon} .
\end{aligned}
$$

As discussed in [1], [3], [4], and [6], for loop bandwidths that are small compared with the reciprocal of the symbol time interval, which is the typical case of interest, $n_{\lambda}(t)$ can be approximated by a delta-correlated process with an equivalent flat (with respect to frequency) PSD. Furthermore, for large loop SNR, it is customary to consider only the value of the PSD at $\lambda=0$, denoted by $N_{0}^{\prime}$. With this in mind, it has been previously shown [1], [3], [4], [6] that $N_{0}^{\prime}$ is computed as

$$
N_{0}^{\prime}=2 T\left[E_{n, s}\left\{\left.e_{k}^{2}\right|_{\lambda=0}\right\}+2 \sum_{m=1}^{\infty} E_{n, s}\left\{\left.e_{k} e_{k+m}\right|_{\lambda=0}\right\}\right] \text {. }
$$

What makes the computation of $N_{0}^{\prime}$ for the NC-LDTTL different from that computed for the coherent LDTTL in [3] is the fact that the noise components in the sine and cosine channels, contained within the error-signal components $e_{s k}$ and $e_{c k}$ that make up the total error signal $e_{k}$, are dependent, since they are derived from the sine and cosine carrier-phase projections of the input signal. After much laborious analysis, the following results are obtained (see the Appendix for the details of the derivation):

$$
\begin{aligned}
E_{n, s}\left\{\left.e_{k}^{2}\right|_{\lambda=0}\right\} & =P^{2} T^{4}\left[\frac{\xi}{4 R_{s}}\left(1+\frac{\xi}{2}+\frac{1}{R_{s}}\right)\right] \\
E_{n, s}\left\{\left.e_{k} e_{k+m}\right|_{\lambda=0}\right\} & = \begin{cases}-P^{2} T^{4} \frac{\xi^{2}}{32 R_{s}}, & m=1 \\
0, & m>1\end{cases}
\end{aligned}
$$

where $R_{s} \triangleq P T / N_{0}$ denotes the detection symbol SNR. Substituting (42) into (41) gives the desired equivalent PSD as

$$
N_{0}^{\prime}=P^{2} T^{5} \frac{\xi}{2 R_{s}}\left(1+\frac{\xi}{4}+\frac{1}{R_{s}}\right) .
$$

Interestingly enough, the result for the coherent LDTTL is given by [3]

$$
N_{0}^{\prime}=P^{2} T^{5} \frac{\xi}{2 R_{s}}\left(1+\frac{\xi}{4}+\frac{1}{2 R_{s}}\right)
$$

although, by comparison, the mathematics employed to arrive at (44) is considerably simpler than that needed to arrive at (43).

\section{Mean-Square Timing-Error Performance}

The mean-square timing error $\sigma_{\lambda}^{2}$ of either the LDTTL or the DTTL is readily computed for a first-order loop filter and large loop SNR conditions. ${ }^{5}$ In particular, linearizing the S-curve to $g(\lambda)=K_{g} \lambda$ and defining the single-sided loop bandwidth by $B_{L}$, we obtain

$$
\sigma_{\lambda}^{2}=\frac{N_{0}^{\prime} B_{L}}{K_{g}^{2}}
$$

where $K_{g}$ is obtained from (39) and $N_{0}^{\prime}$ from (43). Making the appropriate substitutions in (45) gives the results

$$
\left.\sigma_{\lambda}^{2}\right|_{\mathrm{NC}-\mathrm{LDTTL}}=\frac{\xi\left(1+\frac{\xi}{4}+\frac{1}{R_{s}}\right)}{2 \rho\left(1-\frac{\xi}{4}\right)^{2}}
$$

where $\rho \triangleq P / N_{0} B_{L}$ is the so-called phase-locked loop SNR. Using (44) instead of (43) in (45) gives the mean-square timing error for the coherent LDTTL, namely

$$
\left.\sigma_{\lambda}^{2}\right|_{\text {LDTTL }}=\frac{\xi\left(1+\frac{\xi}{4}+\frac{1}{2 R_{s}}\right)}{2 \rho\left(1-\frac{\xi}{4}\right)^{2}} .
$$

\section{TRACKING PERFORMANCE OF THE NC-NLDTTL}

For the NC-NLDTTL illustrated in Fig. 2, analogous to (35) and (36), the error signal components are now given by (48) and (49), shown at the bottom of the next page.

The total error signal $e_{k}$ is now the sum of (48) and (49), which after some trigonometric simplification, becomes (50),

${ }^{5}$ The results are easily generalized to higher order loops following analyses similar to those for tracking loops found in classic textbooks, such as [4] and [5]. Similarly, results for other than high-loop SNR, that depend on statistically modeling the normalized timing error pdf with, say, a Tikhonov distribution [5], can be found by following similar analyses to those in these same references. For the purpose of this paper, namely, to illustrate how the performance of coherent and noncoherent versions of the linear and nonlinear DTTL can differ as a function of symbol SNR, the results for the first-order loop, high loop SNR case treated here are sufficient. 


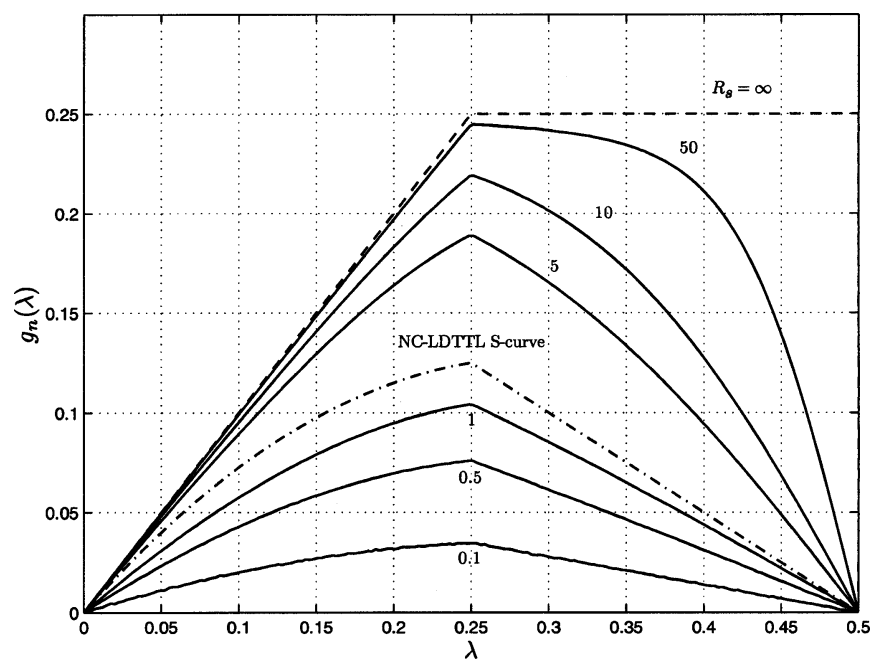

Fig. 3. NC-NLDTTL S-curve, $\xi=0.5$.

shown at the bottom of the page, which is clearly independent of the unknown carrier phase $\theta_{c}$, as desired. To analytically compute even just the $\mathrm{S}$-curve, much less the equivalent PSD, is now a daunting, if not impossible, task. Thus, in order to determine the tracking performance of this scheme, we shall have to turn to results obtained from computer simulations. Before doing this,

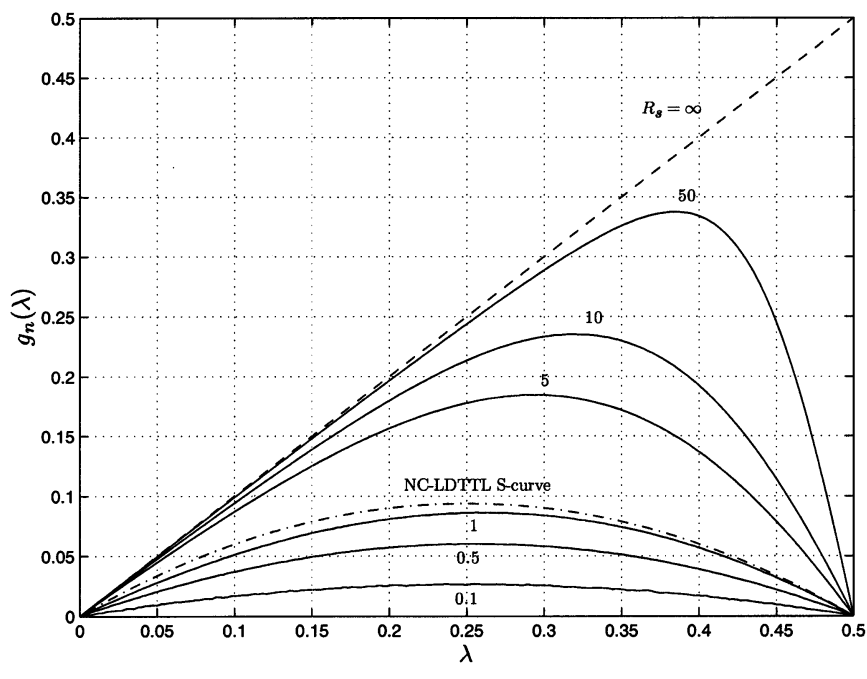

Fig. 4. NC-NLDTTL S-curve, $\xi=1.0$.

however, we do note that in the absence of no noise (i.e., in the limit of infinite SNR), the error signal of (50) becomes

$$
e_{k}=b_{k} \frac{\operatorname{sgn} c_{k}-\operatorname{sgn} c_{k+1}}{2}
$$

which is the exact same result as for the coherent conventional (nonlinear) DTTL, and thus, one can anticipate that in the limit

$$
\begin{aligned}
e_{c k}= & Y_{c k} \times \frac{1}{2}\left[\frac{X_{c k}}{\sqrt{X_{c k}^{2}+X_{s k}^{2}}}-\frac{X_{c, k+1}}{\sqrt{X_{c, k+1}^{2}+X_{s, k+1}^{2}}}\right] \\
= & \left(b_{k} \cos \theta_{c}+\left(M_{c k}^{\prime}+N_{c, k+1}^{\prime}\right) \cos \theta_{c}-\left(M_{s k}^{\prime}+N_{s, k+1}^{\prime}\right) \sin \theta_{c}\right) \\
& \times \frac{1}{2}\left[\frac{\left(c_{k}+N_{c k}+M_{c k}\right) \cos \theta_{c}-\left(N_{s k}+M_{s k}\right) \sin \theta_{c}}{\sqrt{\left(c_{k}+N_{c k}+M_{c k}\right)^{2}+\left(N_{s k}+M_{s k}\right)^{2}}}-\frac{\left(c_{k+1}+N_{c, k+1}+M_{c, k+1}\right) \cos \theta_{c}-\left(N_{s, k+1}+M_{s, k+1}\right) \sin \theta_{c}}{\sqrt{\left(c_{k+1}+N_{c, k+1}+M_{c, k+1}\right)^{2}+\left(N_{s, k+1}+M_{s, k+1}\right)^{2}}}\right]
\end{aligned}
$$

$$
\begin{aligned}
e_{s k}= & Y_{s k} \times \frac{1}{2}\left[\frac{X_{s k}}{\sqrt{X_{c k}^{2}+X_{s k}^{2}}}-\frac{X_{s, k+1}}{\sqrt{X_{c, k+1}^{2}+X_{s, k+1}^{2}}}\right] \\
= & \left(b_{k} \sin \theta_{c}+\left(M_{c k}^{\prime}+N_{c, k+1}^{\prime}\right) \sin \theta_{c}+\left(M_{s k}^{\prime}+N_{s, k+1}^{\prime}\right) \cos \theta_{c}\right) \\
& \times \frac{1}{2}\left[\frac{\left(c_{k}+N_{c k}+M_{c k}\right) \sin \theta_{c}+\left(N_{s k}+M_{s k}\right) \cos \theta_{c}}{\sqrt{\left(c_{k}+N_{c k}+M_{c k}\right)^{2}+\left(N_{s k}+M_{s k}\right)^{2}}}-\frac{\left(c_{k+1}+N_{c, k+1}+M_{c, k+1}\right) \sin \theta_{c}+\left(N_{s, k+1}+M_{s, k+1}\right) \cos \theta_{c}}{\sqrt{\left(c_{k+1}+N_{c, k+1}+M_{c, k+1}\right)^{2}+\left(N_{s, k+1}+M_{s, k+1}\right)^{2}}}\right]
\end{aligned}
$$

$$
\begin{aligned}
e_{k}= & \frac{1}{2}\left(b_{k}+M_{c k}^{\prime}+N_{c, k+1}^{\prime}\right) \\
& \times\left[\frac{c_{k}+N_{c k}+M_{c k}}{\sqrt{\left(c_{k}+N_{c k}+M_{c k}\right)^{2}+\left(N_{s k}+M_{s k}\right)^{2}}}-\frac{c_{k+1}+N_{c, k+1}+M_{c, k+1}}{\sqrt{\left(c_{k+1}+N_{c, k+1}+M_{c, k+1}\right)^{2}+\left(N_{s, k+1}+M_{s, k+1}\right)^{2}}}\right] \\
& +\frac{1}{2}\left(M_{s k}^{\prime}+N_{s, k+1}^{\prime}\right) \\
& \times\left[\frac{N_{s k}+M_{s k}}{\sqrt{\left(c_{k}+N_{c k}+M_{c k}\right)^{2}+\left(N_{s k}+M_{s k}\right)^{2}}}-\frac{N_{s, k+1}+M_{s, k+1}}{\sqrt{\left(c_{k+1}+N_{c, k+1}+M_{c, k+1}\right)^{2}+\left(N_{s, k+1}+M_{s, k+1}\right)^{2}}}\right]
\end{aligned}
$$




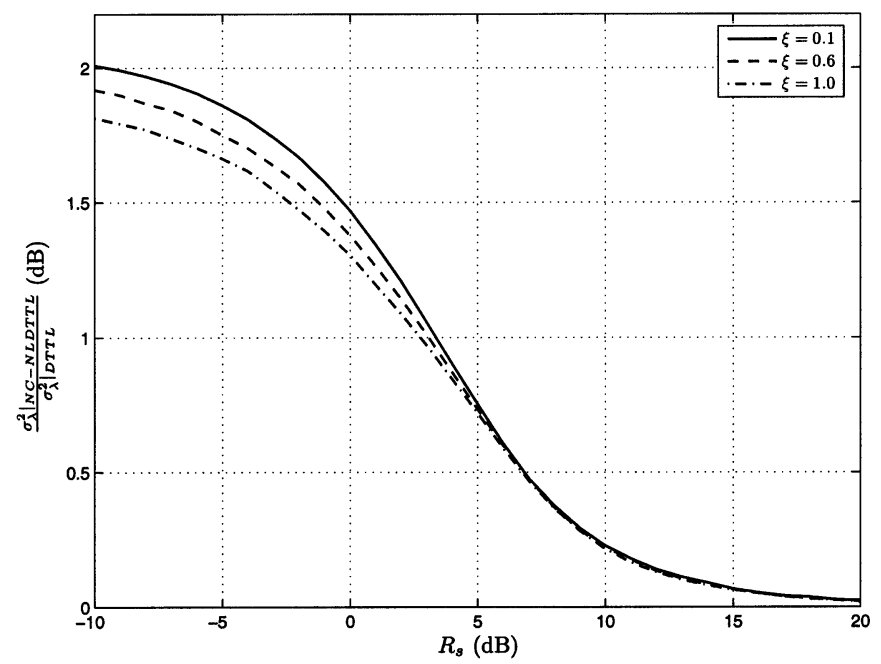

Fig. 5. Ratio of the mean-square timing error for the NC-NLDTTL to that of the coherent (nonlinear) DTTL with window width $(\xi)$ as a parameter.

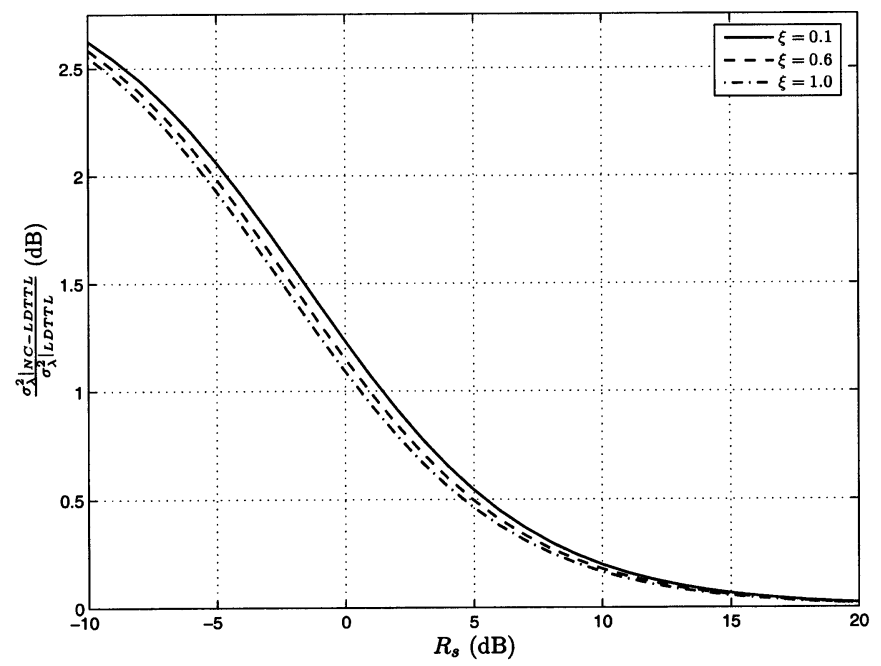

Fig. 6. Ratio of the mean-square timing error for the NC-LDTTL to that of the coherent LDTTL with window width $(\xi)$ as a parameter.

of large SNR, the noncoherent scheme should suffer little or no performance penalty relative to the coherent one.

Figs. 3 and 4 are illustrations of the normalized S-curves for the NC-NLDTTL obtained by computer simulation for two different values of normalized window width and a variety of SNR values. Also superimposed on these results are the corresponding S-curves for the NC-LDTTL, as obtained from (38), which, as previously mentioned, are independent of SNR.

To demonstrate the performance tradeoff of the noncoherent versus the coherent DTTL schemes as a function of SNR, Figs. 5 and 6 plot the mean-square timing error ratios $\left.\sigma_{\lambda}^{2}\right|_{\text {NC-NLDTTL }} /\left.\sigma_{\lambda}^{2}\right|_{\text {DTTL }}$ and $\left.\sigma_{\lambda}^{2}\right|_{\text {NC-LDTTL }} /\left.\sigma_{\lambda}^{2}\right|_{\text {LDTTL }}$, respectively, in decibels, versus SNR in decibels for three different values of normalized window width $\xi$. For the first of the two ratios, the variance $\left.\sigma_{\lambda}^{2}\right|_{\text {NC-NLDTTL }}$ is obtained by computer simulation and then divided by the variance of the

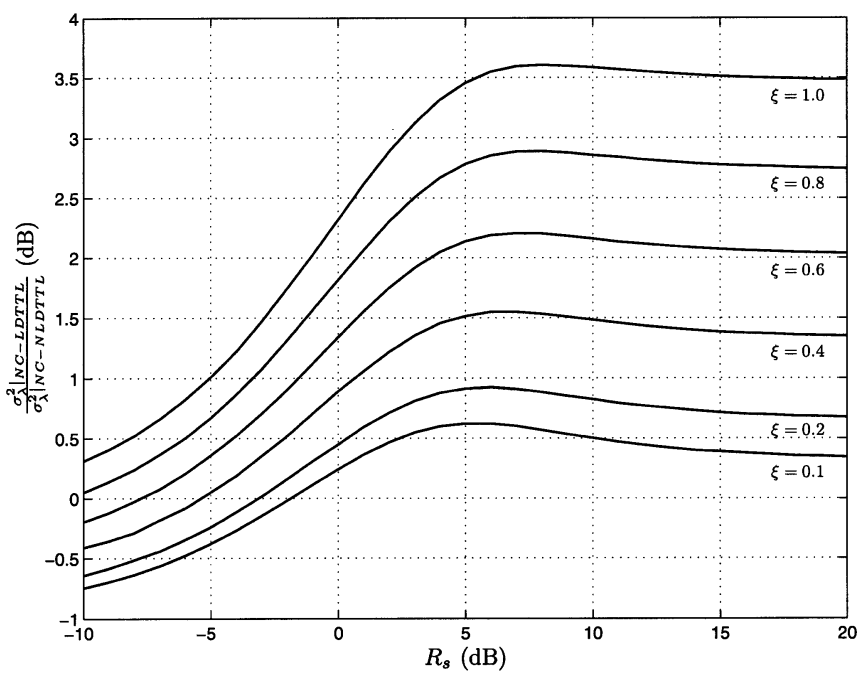

Fig. 7. Ratio of the mean-square timing error for the NC-LDTTL to that of the NC-NLDTTL with window width $(\xi)$ as a parameter.

conventional (nonlinear) DTTL obtained from the results in [1] and $[6]$ as

$$
=\frac{\xi\left(1+\frac{\xi R_{s}}{2}-\frac{\xi}{2}\left[\frac{1}{\sqrt{\pi}} \exp \left(-R_{s}\right)+\sqrt{R_{s}} \operatorname{erf} \sqrt{R_{s}}\right]^{2}\right)}{2 \rho\left(\operatorname{erf} \sqrt{R_{s}}-\frac{\xi}{2} \sqrt{\frac{R_{s}}{\pi}} \exp \left(-R_{s}\right)\right)^{2}} .
$$

The second of the two ratios is simply obtained from the division of (46) by (47). In both cases, we observe that, as expected, the noncoherent and coherent performances approach each other as the SNR gets large (i.e., the above variance ratios approach unity or $0 \mathrm{~dB}$ ). In the limit of infinitesimally small SNR, the noncoherent schemes pay a performance penalty with respect to the coherent schemes, which in the linear case is easily computed from (46) and (47) to be $3 \mathrm{~dB}$, while in the nonlinear case, appears to be somewhat less and mildly dependent on the window width. Finally, a comparison between the noncoherent linear and nonlinear DTTL performances is illustrated in Fig. 7, where the ratio of $\left.\sigma_{\lambda}^{2}\right|_{\text {NC-LDTTL }}$ to $\left.\sigma_{\lambda}^{2}\right|_{\text {NC-NLDTTL }}$ in decibels is plotted, versus SNR in decibels for a variety of different window widths. Analogous to a similar plot for the coherent DTTL schemes in [3], for each window width, there exists a crossover point at which the variance ratio equals unity (or, equivalently, $0 \mathrm{~dB}$ ), indicating the value of SNR that separates the SNR regions where one scheme is preferable over the other.

\section{CONCLUSIONS}

Starting with the MAP estimation of the symbol sync approach, it has been shown how to modify a well-known form of coherent symbol synchronizer, namely, the DTTL, to allow its operation in the absence of carrier-phase information, i.e., as a so-called noncoherent symbol sync loop. By employing such a noncoherent scheme, one can eliminate the need for iteration between the carrier and symbol sync functions, as typically takes place in receivers that more commonly perform carrier tracking and acquisition prior to symbol timing. The 
performance of both the linear and nonlinear versions of this NC-DTTL were obtained by a combination of analysis and simulation, and compared with that of the corresponding coherent DTTLs. It was shown that the differences in performance (as measured by mean-square timing error) between the coherent and noncoherent DTTLs is relatively insensitive to the window width of the loop. Furthermore, the performance of NC-DTTLs approaches that of their coherent counterparts at high SNR, whereas at low SNR values of interest, the penalty is on the order of 1.5-2 dB.

\section{APPENDIX}

DERIVATION OF (41)

We start by deriving the first of the two results given in (41). Summing (35) and (36) and simplifying the trigonometry gives

$e_{k}=\frac{1}{2}\left[b_{k}\left(c_{k}-c_{k+1}\right)-b_{k}\left(\nu_{s k}-\nu_{s, k+1}\right)-\left(c_{k}-c_{k+1}\right) \mu_{s k}\right.$
$\left.+\mu_{c k}\left(\nu_{c k}-\nu_{c, k+1}\right)+\mu_{s k}\left(\nu_{s k}-\nu_{s, k+1}\right)\right]$

which is clearly independent of the carrier-phase error, as desired. Squaring the error signal in (53), averaging over the signal and noise distributions, and then evaluating the result at $\lambda=0$ gives

$$
\begin{aligned}
E_{n, s}\left\{\left.e_{k}^{2}\right|_{\lambda=0}\right\} & \frac{1}{4}\left[E_{s}\left\{\left.b_{k}^{2}\left(c_{k}-c_{k+1}\right)^{2}\right|_{\lambda=0}\right\}\right. \\
& +E_{s}\left\{\left.b_{k}^{2}\right|_{\lambda=0}\right\} E_{n}\left\{\left(\nu_{s k}-\nu_{s, k+1}\right)^{2}\right\} \\
& +E_{s}\left\{\left.\left(c_{k}-c_{k+1}\right)^{2}\right|_{\lambda=0}\right\} E_{n}\left\{\left.\mu_{s k}^{2}\right|_{\lambda=0}\right\} \\
& +E_{n}\left\{\left[\mu_{c k}\left(\nu_{c k}-\nu_{c, k+1}\right)\right]^{2}\right\} \\
& \left.+E_{n}\left\{\left[\mu_{s k}\left(\nu_{s k}-\nu_{s, k+1}\right)\right]^{2}\right\}\right] .
\end{aligned}
$$

Next, we proceed to evaluate each term in (54) one by one.

$$
\begin{aligned}
E_{s} & \left\{b _ { k } ^ { 2 } \left(c_{k}-\frac{\left.\left.c_{k+1}\right)\left.^{2}\right|_{\lambda=0}\right\}}{\left[d_{k-1}\left(\frac{\xi}{2}\right)+d_{k}\left(\frac{\xi}{2}\right)\right]^{2}\left[d_{k-1}-d_{k}\right]^{2}}\right.\right. \\
& =P^{2} T^{4} \\
& =P^{2} T^{4}\left(\frac{\xi}{2}\right)^{2} \overline{\left(2+2 d_{k} d_{k-1}\right)\left(2-2 d_{k} d_{k-1}\right)} \\
& =P^{2} T^{4}\left(\frac{\xi}{2}\right)^{2}\left(4-4 \overline{d_{k-1}^{2} d_{k}^{2}}\right)=0 \\
E_{s} & \left\{\left.b_{k}^{2}\right|_{\lambda=0}\right\} E_{n}\left\{\left(\nu_{s k}-\nu_{s, k+1}\right)^{2}\right\} \\
& =P T^{2}\left(\frac{\xi}{2}\right)^{2} \overline{\left(d_{k-1}+d_{k}\right)^{2}} E_{n}\left\{\nu_{s k}^{2}+\nu_{s, k+1}^{2}\right\} \\
& =\frac{1}{2} P N_{0} T^{3} \xi^{2} \\
E_{s} & \left\{\left.\left(c_{k+1}-c_{k 1}\right)^{2}\right|_{\lambda=0}\right\} E_{n}\left\{\left.\mu_{s k}^{2}\right|_{\lambda=0}\right\} \\
& =P T^{2} \overline{\left(d_{k}-d_{k-1}\right)^{2}} E_{n}\left\{\left.\mu_{s k}^{2}\right|_{\lambda=0}\right\} \\
& =P N_{0} T^{3} \xi \\
E_{n} & \left\{\left[\mu_{c k}\left(\nu_{c k}-\nu_{c, k+1}\right)\right]^{2}\right\} \\
& =E_{n}\left\{\left(\mu_{c k} \nu_{c k}\right)^{2}+\left(\mu_{c k} \nu_{c, k+1}\right)^{2}-2 \mu_{c k}^{2} \nu_{c k} \nu_{c, k+1}\right\}
\end{aligned}
$$

where making use of the properties of higher Gaussian moments, well as the noise correlation properties of $\mu_{c k}$ and $\nu_{c k}$, previously given in Section III, we get

$$
\begin{aligned}
E_{n}\left\{\left(\mu_{c k} \nu_{c k}\right)^{2}\right\} & =E_{n}\left\{\left(\mu_{c k} \nu_{c, k+1}\right)^{2}\right\} \\
& =\left(N_{0} T\right)^{2} \frac{\xi}{4}\left(1+\frac{\xi}{2}\right) \\
E_{n}\left\{\mu_{c k}^{2} \nu_{c k} \nu_{c, k+1}\right\} & =\left(N_{0} T\right)^{2} \frac{\xi^{2}}{8}
\end{aligned}
$$

and thus, we arrive at the simple result

$$
E_{n}\left\{\left[\mu_{c k}\left(\nu_{c k}-\nu_{c, k+1}\right)\right]^{2}\right\}=\left(N_{0} T\right)^{2} \frac{\xi}{2}
$$

Furthermore

$$
E_{n}\left\{\left[\mu_{s k}\left(\nu_{s k}-\nu_{s, k+1}\right)\right]^{2}\right\}=E_{n}\left\{\left[\mu_{c k}\left(\nu_{c k}-\nu_{c, k+1}\right)\right]^{2}\right\}
$$

Finally, substituting (55)-(57) together with (60) and (61) in (54), and recalling that $R_{s}=P T / N_{0}$ gives the desired result

$$
E_{n, s}\left\{\left.e_{k=0}^{2}\right|_{\lambda=}\right\}=P^{2} T^{4}\left[\frac{\xi}{4 R_{s}}\left(1+\frac{\xi}{2}+\frac{1}{R_{s}}\right)\right] .
$$

The second term in (41) is derived by a similar procedure. In particular, analogous to (54), we now have

$$
\begin{aligned}
& E_{n, s}\left\{\left.e_{k} e_{k+1}\right|_{\lambda=0}\right\} \\
& \begin{aligned}
\frac{1}{4}[ & E_{s}\left\{\left.b_{k} b_{k+1}\left(c_{k}-c_{k+1}\right)\left(c_{k+1}-c_{k+2}\right)\right|_{\lambda=0}\right\} \\
& +E_{s}\left\{\left.b_{k} b_{k+1}\right|_{\lambda=0}\right\} E_{n}\left\{\left(\nu_{s k}-\nu_{s, k+1}\right)\left(\nu_{s, k+1}-\nu_{s, k+2}\right)\right\} \\
& +E_{n}\left\{\mu_{c k} \mu_{c, k+1}\left(\nu_{c k}-\nu_{c, k+1}\right)\left(\nu_{c, k+1}-\nu_{c, k+2}\right)\right\} \\
& \left.+E_{n}\left\{\mu_{s k} \mu_{s, k+1}\left(\nu_{s k}-\nu_{s, k+1}\right)\left(\nu_{s, k+1}-\nu_{s, k+2}\right)\right\}\right] .
\end{aligned}
\end{aligned}
$$

Next, evaluating each term in (63), but this time omitting the details, we have

$$
\begin{aligned}
& E_{s}\left\{\left.b_{k} b_{k+1}\left(c_{k}-c_{k+1}\right)\left(c_{k+1}-c_{k+2}\right)\right|_{\lambda=0}\right\}=0 \\
& E_{s}\left\{\left.b_{k} b_{k+1}\right|_{\lambda=0}\right\} E_{n}\left\{\left(\nu_{s k}-\nu_{s, k+1}\right)\left(\nu_{s, k+1}-\nu_{s, k+2}\right)\right\} \\
& \quad=-P^{2} T^{4} \frac{\xi^{2}}{8 R_{s}} \\
& E_{n}\left\{\mu_{c k} \mu_{c, k+1}\left(\nu_{c k}-\nu_{c, k+1}\right)\left(\nu_{c, k+1}-\nu_{c, k+2}\right)\right\} \\
& \quad=E_{n}\left\{\mu_{s k} \mu_{s, k+1}\left(\nu_{s k}-\nu_{s, k+1}\right)\left(\nu_{s, k+1}-\nu_{s, k+2}\right)\right\} \\
& \quad=0 .
\end{aligned}
$$

Thus, substituting (64)-(66) in (63) gives the desired result

$$
E_{n, s}\left\{\left.e_{k} e_{k+1}\right|_{\lambda=0}\right\}=-P^{2} T^{4} \frac{\xi^{2}}{32 R_{s}}
$$

\section{REFERENCES}

[1] M. K. Simon, "An analysis of the steady-state phase noise performance of a digital data-transition tracking loop," in Proc. Int. Conf. Commun., Boulder, CO, Jun. 1969, pp. 20-9-20-15.

[2] S. Haykin, Communication Systems, 4th ed. New York: Wiley, 2001. 
[3] M. K. Simon, A tracking performance comparison of the conventional data transition tracking loop (DTTL) with the linear data transition tracking loop (LDTTL) Jet Propulsion Lab., Pasadena, CA, IPN Prog. Rep. 42-162, Aug. 2005.

[4] W. C. Lindsey and M. K. Simon, Telecommunication Systems Engineering. Englewood Cliffs, NJ: Prentice-Hall, 1973.

[5] W. C. Lindsey, Synchonization Systems in Communication and Control. Englewood Cliffs, NJ: Prentice-Hall, 1972.

[6] M. K. Simon, An analysis of the steady-state phase noise performance of a digital data-transition tracking loop Jet Propulsion Lab., Pasadena, CA, JPL Tech. Rep. 900-222, Nov. 1968.

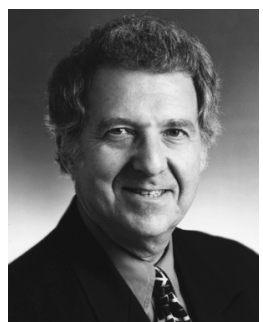

Marvin K. Simon (S'60-M'66-SM'75-F'78 -LF'05) is currently a Principal Scientist at the Jet Propulsion Laboratory (JPL), California Institute of Technology (Caltech), Pasadena, where for the last 37 years he has performed research as applied to the design of NASA's deep-space and near-earth missions, and which has resulted in the issuance of 9 U.S. patents, 26 NASA Tech Briefs, and 4 NASA Space Act awards. He is known as an internationally acclaimed authority on the subject of digital communications with particular emphasis in the disciplines of modulation and demodulation, synchronization techniques for space, satellite, and radio communications, trellis-coded modulation, spread spectrum and multiple access communications, and communication over fading channels. He has also held a joint appointment with the Electrical Engineering Department at Caltech.

He has published over 200 papers on the above subjects and is coauthor of 11 textbooks, including Telecommunication Systems Engineering (Englewood Cliffs, NJ: Prentice-Hall, 1973, and New York: Dover Press, 1991), PhaseLocked Loops and Their Application (New York: IEEE Press, 1978), Spread Spectrum Communications, Vols. I, II, and III (Computer Science Press, 1984 and New York: McGraw-Hill, 1994), An Introduction to Trellis Coded Modulation with Applications (New York: MacMillan, 1991), Digital Communication Techniques: Vol. I (Englewood Cliffs, NJ: Prentice-Hall, 1994) and Digital Communication Over Fading Channels: A Unified Approach to Performance Analysis (New York: Wiley, 2000, 2nd ed. 2005), Probability Distributions In- volving Gaussian Random Variables-A Handbookfor Engineers and Scientists (Norwell, MA: Kluwer, 2002), Bandwidth-Efficient Digital Modulation with Application to Deep-Space Communication (New York: Wiley, 2003), and Autonomous Software-Defined Radio Receivers for Deep Space Applications (New York: Wiley, 2006). His work has also appeared in the textbook Deep Space Telecommunication Systems Engineering (New York: Plenum, 1984), and he is coauthor of a chapter entitled "Spread Spectrum Communications" in the $\mathrm{Mo}$ bile Communications Handbook (Boca Raton, FL: CRC Press, 1995), Communications Handbook (Boca Raton, FL: CRC Press, 1997), and the Electrical Engineering Handbook (Boca Raton, FL: CRC Press, 1997). His work has also appeared in the textbook Deep Space Telecommunication Systems Engineering (New York: Plenum, 1984).

Dr. Simon is the corecipient of the 1988 Prize Paper Award in Communications of the IEEE Vehicular Technology Society, and the 1999 Prize Paper Award of the IEEE Vehicular Technology Conference, Amsterdam, The Netherlands. He is a Fellow of the IAE, and winner of a NASA Exceptional Service Medal, a NASA Exceptional Engineering Achievement Medal, the IEEE Edwin $\mathrm{H}$. Armstrong Achievement Award, and most recently, the IEEE Millennium Medal. He is listed as "highly cited" in a study conducted by the ISI Web of Knowledge, with a total of well over 1500 listings.

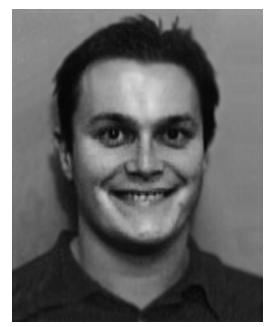

Andre Tkacenko (S'00-M'05) was born in Santa Clara, CA, on February 24, 1977. He received the B.S., M.S., and Ph.D. degrees in electrical engineering from the California Institute of Technology (Caltech), Pasadena, in 1999, 2001, and 2004, respectively.

Currently, he is a member of the Digital Signal Processing Research Group at the Jet Propulsion Laboratory, Pasadena, CA. His research interests include digital signal processing, multirate systems, optimization algorithms, and their applications in digital communications and data compression.

Dr. Tkacenko was awarded the Graduate Division Fellowship from Caltech in 1999. He also received the Charles Wilts Prize in 2004 for outstanding independent research in electrical engineering for his Ph.D. thesis, entitled "Optimization Algorithms for Realizable Signal-Adapted Filter Banks." 